\title{
Validation of the Revised Neuroimaging Radiological Interpretation System For Acute Traumatic Brain Injury in Adult and Pediatric Population
}

\author{
Naresh Kumar Dewangan ${ }^{1} \quad$ Achal Sharma $^{1}$ \\ ${ }^{1}$ Department of Neurosurgery, Sawai Man Singh Medical College \\ and Hospital Jaipur, India
}

Indian J Neurotrauma:2021;18:32-37

\author{
Address for correspondence Naresh Kumar Kumar Dewangan, MS, \\ Department of Neurosurgery, Sawai Man Singh Medical College \\ and Hospital, JLN road, Jaipur 302004, India \\ (e-mail: nd.dewa.mahi@gmail.com).
}

\author{
Abstract \\ Keywords \\ - clinical decision \\ support \\ - computed \\ tomography (CT) \\ - outcome \\ - revised NIRIS \\ - Traumatic Brain \\ Injury (TBI)
}

Aim Our study aimed to validate the revised neuroimaging radiological interpretation system (NIRIS), which would standardize the interpretation of noncontrast head $\mathrm{CT}$ of acute traumatic brain injury (TBI) patient and consolidate imaging finding into ordinal severity categories that would not only inform specific patient management actions but could also be used as a clinical decision support tool.

Methods We retrospectively studied dispositions and their outcomes of consecutive patients brought to the Sawai Man Singh Hospital Trauma Centre, Jaipur, India, by any means of transport and who underwent a noncontrast CT scan for suspected TBI between April and December 2018.

Results The revised NIRIS correctly predicted disposition and outcome in $62.9 \%$ (750/1192) of patients. After excluding patients with OMEI (other major extracranial injuries) and OMII (other major intracranial injuries), a correct prediction was observed in $88.3 \%(670 / 758)$ of patients. After excluding OMEI and OMII, the predictability of revised NIRIS in the adult population is $87.6 \%(446 / 509)$, while predictability in the pediatric population is $92.1 \%$ (224/249).

Conclusion Revised NIRIS is a good tool for predicting patient dispositions, to specific management categories, and outcomes in TBI patients after noncontrast CT head.

\section{Introduction}

Traumatic brain injury (TBI) is a complex multifaceted condition. It is estimated that nearly 1.5 to 2 million persons are injured and 1 million succumb to death every year in India. ${ }^{1}$ An estimated 10 million people worldwide are affected every year by new acute TBI events. ${ }^{2}$ There are many classifications for triage and prediction of mortality in TBI patients. Glasgow coma scale (GCS), which is based on clinical characteristics, stratifies TBI severity, while Marshall ${ }^{3}$ and Rotterdam ${ }^{4}$ scoring are based on radiological classification to predict the mortality in moderate-to-severe TBI., ${ }^{5,6}$ Patients with identical GCS scores were found to have quite different TBI injuries in several clinical trials, illustrating the limited ability of GCS to stratify TBI patients in terms of the pathophysiology of their injury. ${ }^{7}$ Neuroimaging can detect and characterize the presence and extent of brain injury, and plays an important role to stratify and manage TBI patients. ${ }^{8}$

Recently, Wintermark et $\mathrm{al}^{9}$ proposed the neuroimaging radiological interpretation system (NIRIS) for TBI patients, which would standardize the interpretation of noncontrast head $\mathrm{CT}$ and consolidate imaging finding into ordinal severity categories that would not only inform specific patient management actions but could also be used as a clinical decision support tool. The NIRIS is an outcome-based rather than an experience-driven system. According to NIRIS, patients are classified into five mutually exclusive categories: 0discharge from emergency department; 1 -follow-up brain imaging and/or admission; 2-admission to advanced care published online February 4, 2021
DOI https://doi.org/ $10.1055 / \mathrm{s}-0040-1717210$ ISSN 0973-0508.
(C)2021. Neurotrauma Society of India.

This is an open access article published by Thieme under the terms of the Creative Commons Attribution-NonDerivative-NonCommercial-License, permitting copying and reproduction so long as the original work is given appropriate credit. Contents may not be used for commercial purposes, or adapted, remixed, transformed or built upon. (https://creativecommons.org/licenses/by-nc-nd/4.0/).

Thieme Medical and Scientific Publishers Pvt. Ltd. A-12, 2nd Floor, Sector 2, Noida-201301 UP, India 
unit; 3-neurosurgical procedure; 4-death up to 6 months after TBI. When compared with Marshall and Rotterdam scoring system, NIRIS performed similarly in terms of death prediction but was superior in terms of predicting specific patient care actions.

Zhou et $\mathrm{al}^{10}$ successfully validated the NIRIS and proposed the revised NIRIS system (-Table $\mathbf{1}$ ), which predicted TBI patient disposition and outcome with $91.2 \%$ accuracy after excluding patients with other major extracranial traumatic and intracranial nontraumatic injuries.

Recently, Hui Chen et al ${ }^{11}$ characterized the demographics, clinical, and imaging findings and outcomes of TBI patients in each of the NIRIS categories. According to Chen at el, there is a significant difference in NIRIS categories that were observed for all imaging, in agreement with the definition of different NIRIS categories.

The goal of our study was to access the performance of the revised NIRIS system in the Indian population and access the predictability of revised NIRIS in pediatric and adult populations separately.

\section{Methods}

Study design: We retrospectively included consecutive patients who were brought to the Sawai Man Singh Hospital Trauma Centre, Jaipur, India, by any means of transport and who underwent noncontrast CT scans for suspected TBI between April to December 2018. Patients with penetrating brain injuries and gunshot injuries were excluded from our study.

Demographics and all clinical variables of patients are extracted from our institution's medical record department, including age, sex, mechanism of injury, GCS, status at discharge, other major extracranial injuries (OMEI), and other major intracranial injuries (OMII).

The noncontrast CT imaging finding includes the presence/absence of skull fracture, pneumocephalus, hemorrhage, mass effect, and brain parenchymal injuries. Besides, we quantified the volume of epidural, subdural, and parenchymal hematomas and contusions, as well as the amount of midline shift. The volumes of these hematomas and contusions were calculated as their maximal length multiplied by their maximal width multiplied by the number of slices, as they could be seen on multiplying by the slice thickness and dividing by 2 . If a patient presented with several hematomas or contusions, we summed up their volumes to come up with a total volume of hematomas and contusions. We quantified the amount of subarachnoid hemorrhage, intraventricular hemorrhage, brain edema/swelling, cisternal compression and hydrocephalus using ordinal scales ( - Table $\mathbf{3}$ ).

\section{Results}

Study population: 1192 patients (age $47.3 \pm 18.7$ years; $34.8 \%$ female), of which $68.3 \%$ were adults and $31.7 \%$ were of pediatric age (age $\leq 15$ years), were studied retrospectively.

Table 1 The revised NIRIS ${ }^{10}$

\begin{tabular}{|c|c|c|}
\hline Category & Definition & Patient management action \\
\hline NIRIS 0 & No abnormal findings & Discharge from the ED \\
\hline NIRIS 1 & $\begin{array}{l}\text { - Fracture } \pm \\
\text { - Pneumocephalus } \\
\text { - Epidural hematoma, subdural hematoma, parenchy- } \\
\text { mal hematoma or parenchymal contusion }<0.5 \mathrm{~mL} \pm \\
\text { - Subarachnoid hemorrhage }\end{array}$ & Follow up brain imaging and /or admit for observation \\
\hline NIRIS 2 & $\begin{array}{l}\text { - Epidural hematoma, subdural hematoma, parenchy- } \\
\text { mal hematoma or parenchymal contusion > } 0.5 \mathrm{~mL} \pm \\
\text { - Diffuse axonal injury } \pm \\
\text { - Intraventricular hemorrhage } \pm \\
\text { - Mild or moderate hydrocephalus } \pm \\
\text { - Midline shift } 0-5 \mathrm{~mm}\end{array}$ & Admit to a more advanced care unit \\
\hline NIRIS 3 & $\begin{array}{l}\text { - Epidural hematoma, parenchymal hematoma, paren- } \\
\text { chymal contusion }>15 \mathrm{~mL} \pm \\
\text { - Subdural hematoma }>50 \mathrm{~mL} \pm \\
\text { - Midline shift }>5 \mathrm{~mm} \pm \\
\text { - Focal herniation }\end{array}$ & $\begin{array}{l}\text { Consider neurosurgical procedure (ventricular drain, burr } \\
\text { hole, craniotomy/craniectomy, surgical drainage/evacua- } \\
\text { tion of the hematoma }\end{array}$ \\
\hline NIRIS 4 & $\begin{array}{l}\text { - Epidural hematoma, parenchymal hematoma, paren- } \\
\text { chymal contusion } \geq 20 \mathrm{~mL} \pm \\
\text { - Subdural hematoma }>200 \mathrm{~mL} \\
\text { - Severe hydrocephalus } \pm \\
\text { - Midline shift }>10 \mathrm{~mm} \pm \\
\text { - Diffuse herniation } \\
\text { - Duret hemorrhage }\end{array}$ & High-risk of TBI-related death \\
\hline
\end{tabular}

Abbreviations: ED, emergency department; NIRIS, neuroimaging radiological interpretation system; TBI, traumatic brain injury. 
Table 2 Demographic, clinical and injury severity in this study

\begin{tabular}{|c|c|c|c|c|c|c|}
\hline Demographics & Overall & Discharge & $\begin{array}{l}\text { Follow-up brain } \\
\text { imaging/admission }\end{array}$ & ICU stay & Neurosurgery & Death \\
\hline Number of patients & 1192 & 320 & 285 & 226 & 199 & 162 \\
\hline $\begin{array}{l}\text { Age, mean } \pm S D \text { in } \\
\text { years }\end{array}$ & $47.3 \pm 18.7$ & $39.8 \pm 22.8$ & $42.06 \pm 27.9$ & $51 \pm 20.5$ & $46.4 \pm 21.6$ & $59.4 \pm 19.1$ \\
\hline GCS, median (Q1-Q3) & $14(13-14)$ & $15(15-15)$ & $15(13-15)$ & $\begin{array}{l}9.0 \\
(5.5-12.5)\end{array}$ & $14(13-14)$ & $8(3-12)$ \\
\hline Female sex, $n(\%)$ & $415(34.8)$ & $99(30.8)$ & $98(34.4)$ & $88(38.8)$ & $65(32.5)$ & $65(40.0)$ \\
\hline \multicolumn{7}{|c|}{ Mechanism of injury, $n$ (\%) } \\
\hline Fall from height & $347(29.1)$ & $27(8.4)$ & $83(29.1)$ & $95(42.0)$ & $83(41.7)$ & $59(36.4)$ \\
\hline RTA & $644(54.0)$ & $220(68.7)$ & $145(50.8)$ & $80(35.3)$ & $99(49.7)$ & $100(61.7)$ \\
\hline Assault/violence & $171(14.3)$ & $73(22.8)$ & $52(18.9)$ & $32(14.3)$ & $13(6.3)$ & $1(0.6)$ \\
\hline Unknown/other & $30(2.5)$ & $0(0.0)$ & $5(1.7)$ & $19(8.6)$ & $4(2.0)$ & $2(1.2)$ \\
\hline OMII n (\%) & $87(7.2)$ & $0(0.0)$ & $42(14.6)$ & $22(9.7)$ & $15(7.3)$ & $8(4.9)$ \\
\hline OMEI $n(\%)$ & $339(28.4)$ & $126(39.4)$ & $91(32.1)$ & $26(11.3)$ & $73(36.9)$ & $23(14.0)$ \\
\hline $\begin{array}{l}\text { Secondary intracranial } \\
\text { complications, } n(\%)\end{array}$ & $118(9.8)$ & $0(0.0)$ & $0(0.0)$ & $22(9.6)$ & $36(18.3)$ & $60(36.8)$ \\
\hline $\begin{array}{l}\text { Secondary extracranial } \\
\text { complications, } n(\%)\end{array}$ & $220(18.4)$ & $0(0.0)$ & $0(0.0)$ & $76(33.6)$ & $57(28.7)$ & $87(53.9)$ \\
\hline \multicolumn{7}{|c|}{ Follow-up brain imaging studies, $n$ (\%) } \\
\hline Noncontrast CT head & $484(40.6)$ & $0(0.0)$ & $117(41.0)$ & $120(53.1)$ & $188(94.3)$ & $59(36.2)$ \\
\hline $\begin{array}{l}\text { Contrast-enhanced } \\
\text { CT head }\end{array}$ & $19(1.6)$ & $0(0.0)$ & $4(1.4)$ & $8(3.5)$ & $7(3.5)$ & $0(0.0)$ \\
\hline $\begin{array}{l}\text { Intracranial or cervical } \\
\text { CT angiography }\end{array}$ & $105(8.8)$ & $0(0.0)$ & $56(19.6)$ & $30(13.2)$ & $12(6.0)$ & $11(6.7)$ \\
\hline Brain MRI & $75(6.3)$ & $0(0.0)$ & $26(2.1)$ & $38(16.8)$ & $9(4.5)$ & $2(1.2)$ \\
\hline $\begin{array}{l}\text { Intracranial or cervical } \\
\text { MR angiography }\end{array}$ & $11(0.9)$ & $0(0.0)$ & $4(1.4)$ & $5(2.2)$ & $2(1.0)$ & $0(0.0)$ \\
\hline
\end{tabular}

Abbreviations: GCS, Glasgow coma scale; OMEI, other major extracranial injuries; OMII, other major intracranial injuries; RTA, road traffic accidents.

\section{Clinical Data}

The clinical characteristics of our study patients are reported in - Table 2. Road traffic accidents (RTA) is the leading cause (54\%) of TBIs, followed by fall from height $(29.1 \%)$ and violence (14.3\%). As much as $28.4 \%$ of these patients had other major extracranial injuries and $7.2 \%$ had other major intracranial nontraumatic injuries. The GCS score decreased with increasing revised NIRIS category, and it was significantly lower in patients with revised NIRIS category 4 . As much as $40.6 \%$ of patients underwent noncontrast CT of head as follow-up imaging, while $1.6 \%$ underwent contrast CT of head; $8.8 \%$ of these patients had done intracranial/cervical CT angiography and 6.3\% had follow-up brain imaging.

\section{Imaging Results}

The distribution of imaging common data elements in this study population, stratified by the patient outcome, is depicted in - Table 3 . As much as $30.2 \%$ patients had a skull fracture, with $22.5 \%$ sustaining a calvarial fracture and $8.8 \%$ sustaining a skull base fracture, while $70(5.8 \%)$ patients sustained a depressed fracture, of which 30 patients (42.8\%) met the criteria for neurosurgery and got operated. A total of $86(7.2 \%)$ patients were admitted with intraventricular hemorrhage, of which eight (9.3\%) patients were operated with external ventricular drain (EVD). As much as $26.4 \%$ of patients had mass effect, while $14 \%$ of patients had brain herniation.

The performance of the revised NIRIS classification in terms of predicting dispositions and outcomes in the retrospective cohort $(n=1192)$ is depicted in - Table 4 . The revised NIRIS correctly predicted patient dispositions and outcomes in $62.9 \%(750 / 1192)$ of patients. After excluding patients with OMEI and OMII, a correct prediction was observed in 88.3\% (670/758). After excluding OMEI and OMII, the predictability of revised NIRIS in the adult population is $87.6 \%$ (446/509), while predictability in the pediatric population is $92.1 \%(224 / 249)$.

\section{Comparison of Revised NIRIS, Marshall and Rotterdam Scoring System for All Five Outcome Categories}

The outcome for each score level for revised NIRIS, Marshall, and Rotterdam scoring system is outlined in Supplementary 
Table 3 Imaging CDE in this study

\begin{tabular}{|c|c|c|c|c|c|c|}
\hline & Overall & Discharge & $\begin{array}{l}\text { Follow-up brain } \\
\text { imaging/admission }\end{array}$ & ICU stay & Neurosurgery & Death \\
\hline Number of patients & 1192 & 320 & 285 & 226 & 199 & 162 \\
\hline Skull fracture, n (\%) & $361(30.2)$ & $0(0.0)$ & $98(34.3)$ & $81(35.8)$ & $103(51.7)$ & $79(48.7)$ \\
\hline Calvarial fracture & $269(22.5)$ & $0(0.0)$ & $89(31.2)$ & $44(19.4)$ & $87(43.7)$ & $49(30.2)$ \\
\hline Skull base fracture & $106(8.8)$ & $0(0.0)$ & $14(4.9)$ & $29(12.8)$ & $24(12.0)$ & $39(24.0)$ \\
\hline Depressed fracture & $70(5.8)$ & $0(0.0)$ & $09(3.1)$ & 18 (7.9) & $30(15.0)$ & $13(8.0)$ \\
\hline Pneumocephalus n (\%) & $146(12.2)$ & $0(0.0)$ & $39(13.6)$ & $56(24.7)$ & $33(16.5)$ & $18(11.1)$ \\
\hline Hemorrhage n (\%) & $564(47.3)$ & $0(0.0)$ & $100(35.0)$ & $141(62.3)$ & $180(90.4)$ & $143(88.2)$ \\
\hline Epidural hematoma & $184(15.4)$ & $0(0.0)$ & $46(16.1)$ & $44(19.4)$ & $66(33.1)$ & $28(17.2)$ \\
\hline Subdural hematoma & $274(22.9)$ & $0(0.0)$ & $18(6.3)$ & $82(36.2)$ & $72(36.1)$ & $102(62.9)$ \\
\hline $\begin{array}{l}\text { Subarachnoid } \\
\text { hemorrhage }\end{array}$ & $186(15.6)$ & $0(0.0)$ & $37(12.9)$ & $84(37.1)$ & $32(16.0)$ & $33(20.3)$ \\
\hline $\begin{array}{l}\text { Intraventricular } \\
\text { hemorrhage }\end{array}$ & $86(7.2)$ & $0(0.0)$ & $0(0.0)$ & $43(19.0)$ & $8(4.0)$ & $35(21.6)$ \\
\hline $\begin{array}{l}\text { Parenchymal hemat- } \\
\text { oma (including hemor- } \\
\text { rhagic contusions }\end{array}$ & $398(33.3)$ & $0(0.0)$ & $118(41.4)$ & $126(55.7)$ & $58(29.1)$ & $96(59.2)$ \\
\hline Diffuse axonal injury & $68(5.7)$ & $0(0.0)$ & $6(2.1)$ & $56(24.7)$ & $4(2.0)$ & $2(1.2)$ \\
\hline Mass effect, n (\%) & $315(26.4)$ & $0(0.0)$ & $9(3.1)$ & $37(16.3)$ & $171(85.9)$ & $98(60.4)$ \\
\hline $\begin{array}{l}\text { Brain edema/ } \\
\text { swelling }\end{array}$ & $279(23.4)$ & $0(0.0)$ & $12(4.2)$ & $76(33.6)$ & $89(44.7)$ & 102 (62.9) \\
\hline Midline shift & $406(34.0)$ & $0(0.0)$ & $38(13.3)$ & $89(39.3)$ & $170(85.4)$ & $109(67.2)$ \\
\hline $\begin{array}{l}\text { Cisternal } \\
\text { compression }\end{array}$ & $206(17.2)$ & $0(0.0)$ & $14(4.9)$ & $32(14.1)$ & $81(0.40)$ & $79(48.7)$ \\
\hline $\begin{array}{l}\text { Brain herniation/Duret } \\
\text { hemorrhage }\end{array}$ & $167(14.0)$ & $0(0.0)$ & $0(0.0)$ & $18(7.9)$ & $66(0.33)$ & $83(51.2)$ \\
\hline Hydrocephalus & $173(14.5)$ & $0(0.0)$ & $41(14.3)$ & $52(23.0)$ & $41(20.6)$ & $39(24.0)$ \\
\hline Mild & $134(11.2)$ & $0(0.0)$ & $40(14.0)$ & 49 (21.6) & $22(11.0)$ & $23(14.1)$ \\
\hline Moderate & $25(2.0)$ & $0(0.0)$ & $1(0.3)$ & $1(0.4)$ & $11(5.5)$ & $12(7.4)$ \\
\hline Severe & $14(1.1)$ & $0(0.0)$ & $0(0.0)$ & $2(0.8)$ & $8(4.0)$ & $4(2.4)$ \\
\hline $\begin{array}{l}\text { Non hemorrhagic } \\
\text { contusions }\end{array}$ & $67(5.6)$ & $0(0.0)$ & $18(6.3)$ & $24(10.6)$ & $13(6.5)$ & $12(7.4)$ \\
\hline
\end{tabular}

Abbreviation: $\mathrm{CDE}$, common data elements.

Table S1. Marshall scores of 1 and 2 were associated with discharge from the emergency department (ED), hospital admission, and intensive care (ICU) monitoring, but it could not differentiate among these outcomes. Marshall scores of 5 and 6 were associated with the neurosurgical procedure and patient death.

In our study, most of the Rotterdam scores were 2 and 3 , which did not differentiate among the five outcomes.

\section{Extended Glasgow Outcome Scale (GOSE) and Mortality}

Three-month GOSE scores were successfully obtained for 604 patients out of 1192 patients (50.7\%) (Supplementary Tables S2 and S3). Among 604 patients, there were 38 dead patients. The GOSE score was significantly higher $(p<$ 0.0001 ) in revised NIRIS 3 and revised NIRIS 4. Mortality progressively increased with increasing revised NIRIS category. Mortality in patients with OMEI and OMII was significantly higher than those patients without OMEI and OMII in revised NIRIS 0.

\section{Discussion}

In our study, the revised NIRIS correctly predicted patient dispositions and outcomes in 62.9\% (750/1192) of patients. After excluding patients with OMEI and OMII, a correct prediction was observed in $88.3 \%$ (670/758) of patients. After excluding OMEI and OMII, the predictability of revised NIRIS in the adult population was $87.6 \%(446 / 509)$, while predictability in the pediatric population (age $\leq 15$ years) was $92.1 \%$ (224/249). To date, no study has been conducted to get NIRIS predictability for patient disposition in the pediatric population. In the previous study, ${ }^{10}$ revised NIRIS correctly predicted dispositions and outcomes in $60.5 \%$ of patients, and after excluding the patients with OMEI and OMII, a corrected prediction was observed in $91.2 \%$.

Still, the revised NIRIS should be considered for revision, as in our study, 30 patients with depressed skull fracture (those who met the criteria for operability) were kept in the revised NIRIS category 1 . After the inclusion of depressed fracture patients in revised NIRIS category 3 , overall predictability increased from $88.3 \%$ to $92.3 \%$, predictability in 
Table 4 Distribution of patient dispositions and outcomes in our retrospective study

\begin{tabular}{|c|c|c|c|c|c|c|}
\hline NIRIS CT & Discharge & Admission & ICU & Neurosurgery & Death & Total \\
\hline 0 & 320 & 103 & 54 & 0 & 45 & 522 \\
\hline 1 & 0 & 182 & 40 & 32 & 24 & 278 \\
\hline 2 & 0 & 0 & 80 & 10 & 17 & 107 \\
\hline 3 & 0 & 0 & 42 & 133 & 41 & 216 \\
\hline 4 & 0 & 0 & 10 & 24 & 35 & 69 \\
\hline Total & 320 & 285 & 226 & 199 & 162 & 1192 \\
\hline \multicolumn{7}{|c|}{ Study population minus patients with OMEI AND OMII $(n=758)$} \\
\hline 0 & 320 & 03 & 0 & 0 & 0 & 323 \\
\hline 1 & 0 & 130 & 20 & 30 & 0 & 180 \\
\hline 2 & 0 & 0 & 64 & 6 & 5 & 75 \\
\hline 3 & 0 & 0 & 6 & 121 & 8 & 135 \\
\hline 4 & 0 & 0 & 4 & 6 & 35 & 45 \\
\hline Total & 320 & 133 & 94 & 163 & 48 & 758 \\
\hline \multicolumn{7}{|c|}{ Distribution of adult patient dispositions and outcomes in our retrospective study $(n=815)$} \\
\hline 0 & 203 & 62 & 30 & 0 & 34 & 329 \\
\hline 1 & 0 & 135 & 32 & 24 & 20 & 211 \\
\hline 2 & 0 & 0 & 37 & 8 & 7 & 52 \\
\hline 3 & 0 & 0 & 32 & 107 & 31 & 170 \\
\hline 4 & 0 & 0 & 10 & 22 & 21 & 53 \\
\hline Total & 203 & 197 & 141 & 161 & 113 & 815 \\
\hline \multicolumn{7}{|c|}{ Adult population minus patients with OMEI AND OMII $(n=509)$} \\
\hline 0 & 203 & 03 & 0 & 0 & 0 & 206 \\
\hline 1 & 0 & 87 & 12 & 24 & 0 & 123 \\
\hline 2 & 0 & 0 & 34 & 6 & 3 & 43 \\
\hline 3 & 0 & 0 & 2 & 95 & 5 & 102 \\
\hline 4 & 0 & 0 & 4 & 4 & 27 & 35 \\
\hline Total & 203 & 90 & 52 & 129 & 35 & 509 \\
\hline \multicolumn{7}{|c|}{ Distribution of pediatric patient dispositions and outcomes in our retrospective study $(n=377)$} \\
\hline 0 & 117 & 41 & 24 & 0 & 11 & 193 \\
\hline 1 & 0 & 47 & 8 & 8 & 04 & 67 \\
\hline 2 & 0 & 0 & 43 & 2 & 10 & 55 \\
\hline 3 & 0 & 0 & 10 & 26 & 10 & 46 \\
\hline 4 & 0 & 0 & 0 & 2 & 14 & 16 \\
\hline Total & 117 & 88 & 85 & 38 & 49 & 377 \\
\hline \multicolumn{7}{|c|}{ Pediatric population minus patients with OMEI AND OMII $(n=249)$} \\
\hline 0 & 117 & 0 & 0 & 0 & 0 & 117 \\
\hline 1 & 0 & 43 & 8 & 6 & 0 & 57 \\
\hline 2 & 0 & 0 & 30 & 0 & 2 & 32 \\
\hline 3 & 0 & 0 & 4 & 26 & 3 & 33 \\
\hline 4 & 0 & 0 & 0 & 2 & 8 & 10 \\
\hline Total & 117 & 43 & 42 & 34 & 13 & 249 \\
\hline
\end{tabular}

Abbreviations: NIRIS, neuroimaging radiological interpretation system; OMEI, other major extracranial injuries; OMII, other major intracranial injuries. Note: The gray shaded cells are presenting the exact number of disposed of patients in the respective revised NIRIS category.

the adult age group increased from $87.6 \%$ to $92.3 \%$, and predictability increased from $92.1 \%$ to $92.3 \%$ in the pediatric age group. After keeping depressed fracture (those who met the criteria for operability) in revised NIRIS category 3, the predictability of revised NIRIS is similar in the pediatric and adult population. 
TBI is a major cause of morbidity and mortality. Road traffic accidents (RTA), which is a leading cause of TBI in many parts of the world, is expected to become the third-largest cause of global disease burden by $2020 .{ }^{12}$

Some studies focused on clinical characteristics that predict the outcome in TBI patients, including the IMPACT ${ }^{13}$ and TRACT-TBI studies. ${ }^{14}$ These studies have verified the prognostic value of many known predictors associated with worse GOS scores (e.g., age, GCS,,$^{15}$ pupil response, ${ }^{15}$ CT findings, ${ }^{16}$ pre-existing psychiatric conditions, and lower education).

In our study, the most common cause of TBI is RTA and it involves mostly the young population. We found that patients within the category revised NIRIS 0 registered good GCS and GOSE scores at 3 months, and as the grading of revised NIRIS category increases, there is a decrease in the GCS and GOSE scoring of patients. Patients within a higher revised NIRIS category have major intracranial or extracranial injuries with a longer hospital stay, need ICU monitoring, and may die from their injury. In our study, the cause of longer hospital stays, ICU monitoring and mortality is determined not only by severity of TBI but also by other intracranial and extracranial injuries. The extracranial injury explains the high morbidity and mortality in lower revised NIRIS categories like revised NIRIS 0 and revised NIRIS 1.

In a previous study, ${ }^{9}$ as well as our study, revised NIRIS was found to perform similarly to the Rotterdam scale and the Marshall scoring system in terms of predicting survival/death, while revised NIRIS performed better than the Rotterdam and Marshall scoring systems in terms of predicting discharge, admission, follow-up neuroimaging, advanced care unit stay, and neurosurgical procedures.

Our study has several limitations. One of the main limitations is that it is a retrospective study, causing selection and information bias. With retrospective studies, the temporal relationship is frequently difficult to assess. Our study is based on a CT scan only. Our study did not include those patients with TBI who do not fulfill the criteria for CT of head, which may be a cause of selection bias. MRI is done in only a few patients who are suspected of diffuse axonal injury (DAI) and not on ventilatory support. Our institute is a high-volume center and many cases of TBI are categorized as brought dead and those cases are not included in our study.

\section{Conclusion}

CT findings in combination with GCS scoring is likely to provide the best outcome in TBI patient management. We successfully validated the revised NIRIS in the Indian population with adult and pediatric subpopulations. Revised NIRIS is a good tool for predicting patient dispositions, to specific management categories, and outcomes. Still, revised NIRIS requires some correction to improve predictability, and multicentre cross-validation with the help of a prospective study is still required.

\section{Conflict of Interest}

None declared.

\section{References}

1 Gururaj G. Epidemiology of traumatic brain injuries: Indian scenario. Neurol Res 2002;24(1):24-28

2 Feigin VL, Theadom A, Barker-Collo S, et al; BIONIC Study Group. Incidence of traumatic brain injury in New Zealand: a population-based study. Lancet Neurol 2013;12(1):53-64

3 Marshall LF, Marshall SB, Klauber MR, et al. The diagnosis of head injury requires a classification based on computed axial tomography. J Neurotrauma 1992;9(Suppl 1) :S287-S292

4 Maas AI, Hukkelhoven CW, Marshall LF, Steyerberg EW. Prediction of outcome in traumatic brain injury with computed tomographic characteristics: a comparison between the computed tomographic classification and combinations of computed tomographic predictors. Neurosurgery 2005;57(6):1173-1182, discussion 1173-1182

5 Munakomi S, Bhattarai B, Srinivas B, Cherian I. Role of computed tomography scores and findings to predict early death in patients with traumatic brain injury: A reappraisal in a major tertiary care hospital in Nepal. Surg Neurol Int 2016;7:23

6 Mata-Mbemba D, Mugikura S, Nakagawa A, et al. Early CT findings to predict early death in patients with traumatic brain injury: Marshall and Rotterdam CT scoring systems compared in the major academic tertiary care hospital in northeastern Japan. Acad Radiol 2014;21(5):605-611

7 Stead LG, Wijdicks EF, Bhagra A, et al. Validation of a new coma scale, the FOUR score, in the emergency department. Neurocrit Care 2009;10(1):50-54

8 Lingsma HF, Roozenbeek B, Steyerberg EW, Murray GD, Maas AI. Early prognosis in traumatic brain injury: from prophecies to predictions. Lancet Neurol 2010;9(5):543-554

9 Wintermark M, Li Y, Ding VY, et al. Neuroimaging radiological interpretation system for acute traumatic brain injury. J Neurotrauma 2018;35(22):2665-2672

10 Zhou B, Ding VY, Li Y, et al. Validation of the neuroimaging radiological interpretation system for acute traumatic brain injury. J Comput Assist Tomogr 2019;43(5):690-696

11 Chen H, Li Y, Jiang B, et al. Demographics and clinical characteristics of acute traumatic brain injury patients in the different Neuroimaging Radiological Interpretation System (NIRIS) categories. J Neuroradiol 2019 (e-pub ahead of print). Doi: https://doi.org/10.1016/j.neurad.2019.07.002

12 Edge L. Traumatic brain injury: time to end the silence. Lancet Neurol 2010;9(4):331

13 Murray GD, Butcher I, McHugh GS, et al. Multivariable prognostic analysis in traumatic brain injury: results from the IMPACT study. J Neurotrauma 2007;24(2):329-337

14 Lingsma HF, Yue JK, Maas AI, Steyerberg EW, Manley GT; TRACK-TBI Investigators. Outcome prediction after mild and complicated mild traumatic brain injury: external validation of existing models and identification of new predictors using the TRACK-TBI pilot study. J Neurotrauma 2015;32(2):83-94

15 Marmarou A, Lu J, Butcher I, et al. Prognostic value of the Glasgow Coma Scale and pupil reactivity in traumatic brain injury assessed pre-hospital and on enrollment: an IMPACT analysis. J Neurotrauma 2007;24(2):270-280

16 Maas AI, Steyerberg EW, Butcher I, et al. Prognostic value of computerized tomography scan characteristics in traumatic brain injury: results from the IMPACT study. J Neurotrauma 2007;24(2):303-314 